\title{
Síndrome do Entrelaçamento da Artéria Poplítea: um Diagnóstico Diferencial de Claudicação de Membros Inferiores: Contribuição da Ressonância Magnética para Diagnóstico e Avaliação
}

\section{Popliteal Artery Entrapment Syndrome: a Differential Diagnosis in Lower Limbs Claudication: Contribution of Masnetic Resonance Imasing for Diagnosis and Evaluation}

\author{
Marcos Hiroyuki Ikawa ${ }^{1}$, Jamil Natour ${ }^{2}$, Marcelo Guedes Jannini ${ }^{1}$, Leonardo Massamaru Sugawara ${ }^{1}$, \\ Rogério Diniz Takahashi ${ }^{1}$, Artur da Rocha Correa Fernandes ${ }^{1}$
}

\section{INTRODUÇÃO}

A síndrome do entrelaçamento da artéria poplítea é uma entidade pouco comum, mais prevalente em pacientes jovens e atletas, que passam a apresentar claudicação na perna resultante da compressão arterial extrínseca na região da fossa poplítea, por um ventre ou inserção anômala do músculo gastrocnêmio ${ }^{(1,2)}$.

\section{ASPECTOS CLÍNICOS}

A doença arterial poplítea crônica ocorre mais comumente devido à aterosclerose, mas também por outras doenças como embolismo, aneurisma trombosado, compressão extrínseca por cisto poplíteo e doença cística adventícia. Uma causa menos comum, mas que também pode levar a sintomas de insuficiência arterial crônica, é a síndrome do entrelaçamento da artéria poplítea.

Ocorre devido a uma relação anatômica anormal que se desenvolve durante a embriogênese, entre a artéria poplítea e o músculo gastrocnêmio. Diversos tipos de alterações podem levar à síndrome, como trajeto aberrante da artéria poplítea, inserção anômala da cabeça medial do músculo gastrocnêmio, presença de ventre acessório do gastrocnêmio ou de uma banda fibrosa comprimindo a artéria poplítea na fossa poplítea.

A síndrome do entrelaçamento da artéria poplítea usualmente é vista em pacientes jovens, atletas e do sexo masculino, com claudicação progressiva da perna, sendo bilateral em até um terço dos casos. Classicamente há ausência de pulso distal no pé durante a dorsiflexão passiva ou flexão plantar ativa.

Por vezes, indivíduos sem qualquer das anomalias acima citadas, porém com musculatura bem desenvolvida e hipertrofiada que comprime os vasos poplíteos, podem cursar com os mesmos sintomas, desenvolvendo uma síndrome do entrelaçamento funcional ${ }^{(3)}$.

\section{ACHADOS DE IMAGEM}

O ultra-som, especialmente o estudo por Doppler, pode evidenciar alterações hemodinâmicas resultantes da compressão e tem a vantagem de permitir a realização de manobras dinâmicas que simulam a clínica ou potencializem as alterações estenóticas. Entretanto, esse método pode mostrar oclusão arterial em até $59 \%$ de pessoas assintomáticas, ou mesmo nenhuma alteração em pacientes com a síndrome, apresentando, portanto, baixa sensibilidade e especificidade ${ }^{(4)}$.

A angiografia convencional pode caracterizar diversas alterações na artéria poplítea. Rosset et al. demonstraram oclusão em $36 \%$ dos casos, desvios em $24 \%$, ectasia ou aneurisma em $9 \%$ e estenose dinâmica em $32 \%^{(5)}$. Porém não foi possível a diferenciação da estenose por alguma compressão extrínseca daquela causada por fatores ateroscleróticos ou degenerativos (Figura 2).

A ressonância magnética permite melhor estudo da anatomia da fossa poplítea sem uso de radiação ionizante ou contraste iodado endovenoso, com alto contraste

Declaramos a inexistência de conflitos de interesse.

1. Departamento de Diagnóstico por Imagem da Universidade Federal de São Paulo (Unifesp).

2. Disciplina de Reumatologia da Unifesp.

Endereço para correspondência: Artur da Rocha Correa Fernandes, DDI/Unifesp, Rua Botucatu, 740, CEP 04023-900, São Paulo, SP. 
entre estruturas musculares, ósseas, vasculares e planos gordurosos. É a modalidade de imagem que permite, com maior facilidade, identificar o fator causal dessa síndrome. Seqüências spin-eco ou fast spin-eco podem mostrar a perda do "flow-poid" no interior da artéria poplítea devido à trombose ou fluxo lentificado (Figuras 1, 2, 3 e 4). Técnicas angiográficas permitem avaliar o comprometimento vascular durante a flexão dorsal e plantar do pé. E por fim, a utilização da angioressonância com gadolínio endovenoso melhora a detecção de trajetos vasculares na eventual presença de aneurismas ou estenoses distais ${ }^{(1,2,3,4)}$.

Nesta paciente do sexo feminino, 10 anos, com queixa de claudicação na perna direita de longa data, o ultra-som prévio evidenciou deslocamento medial e trombose parcial da artéria poplítea. As imagens de ressonância magnética de 1,5 Tesla, multiplanares com técnica fast spin-eco ponderadas em T1, T2, e gradiente eco com saturação de gordura, evidenciaram inserção femoral anormalmente alta e discretamente lateralizada da cabeça medial do músculo gastrocnêmio. Os vasos poplíteos apresentam trajetos levemente deslocados medialmente, apresentando-se comprimidos contra a região metaepifisária distal do fềmur pouco acima da interlinha articular femorotibial. Nessa região, a artéria poplítea mostrou redução significativa do calibre, alteração da forma e perda do "flow-void", um indicativo de trombose e/ou fluxo lentificado, sendo até mesmo difícil de visualizar em alguns trechos.
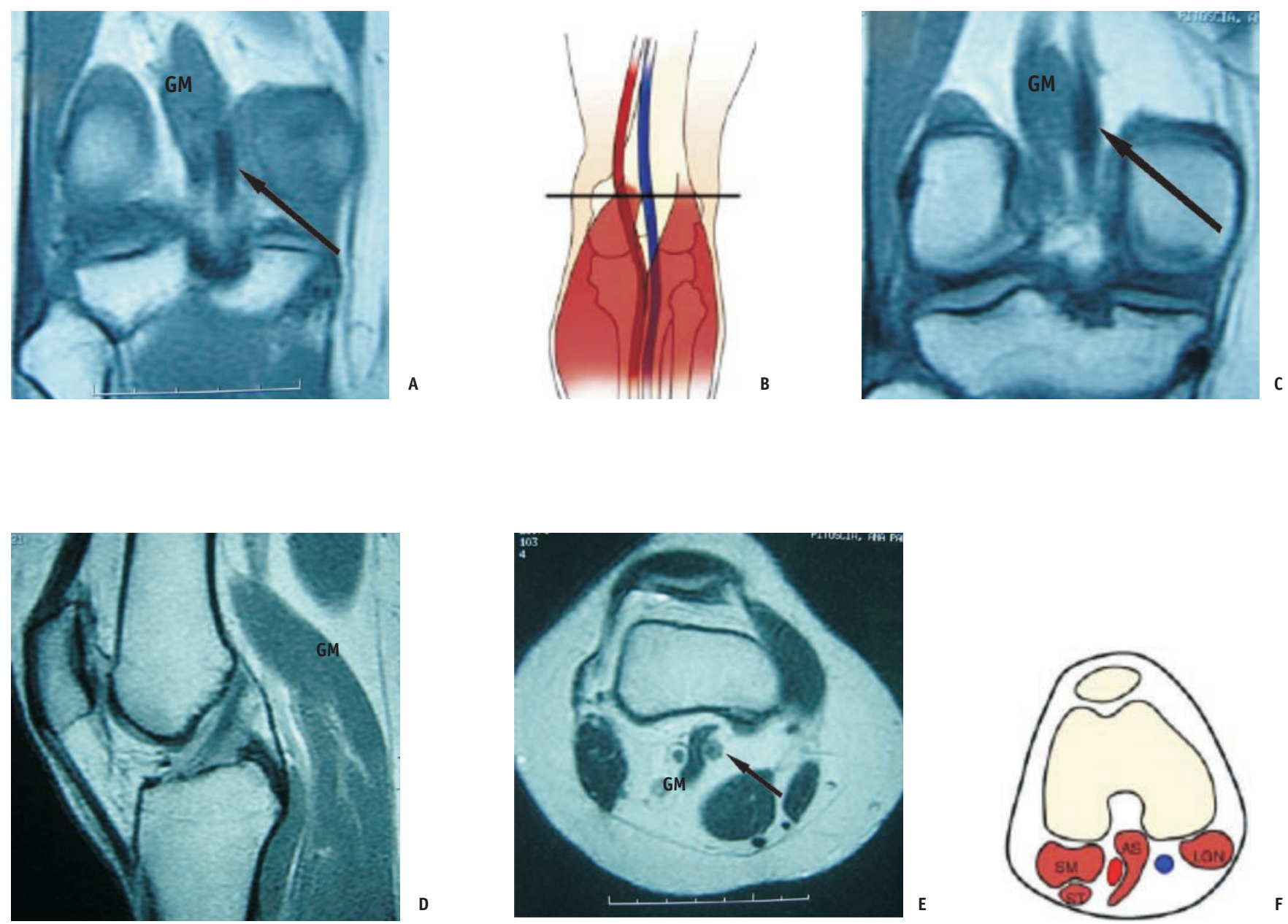

Figura 1 - Paciente de 32 anos, sexo feminino, praticante de corrida com dor na perna há um ano.

Cortes coronais, axial e sagital evidenciando inserção mais alta e lateral do gastrocnêmio medial, comprimindo os vasos poplíteos. Entrelaçamento tipo II segundo Kim e cols. 

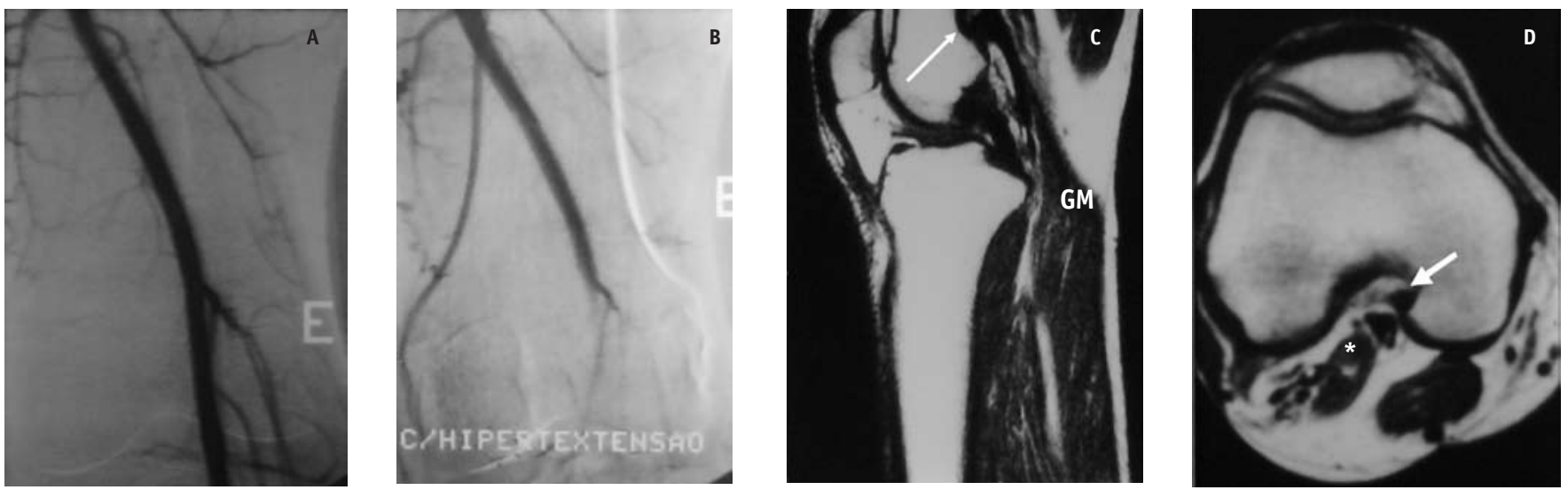

Figura 2 - Arteriografia do membro inferior esquerdo ao nível da artéria poplítea, que apresenta forma e topografia normais em repouso (A). Às manobras dinâmicas (B), observa-se compressão extrínseca com interrupção da passagem do contraste por sua luz. Imagens C e D da ressonância magnética no mesmo paciente mostrando a inserção alta (seta longa em C) e trajeto lateralizado (asterisco em D) do ventre medial do gastrocnêmio (GM). Seta curta em D: Vasos poplíteos.
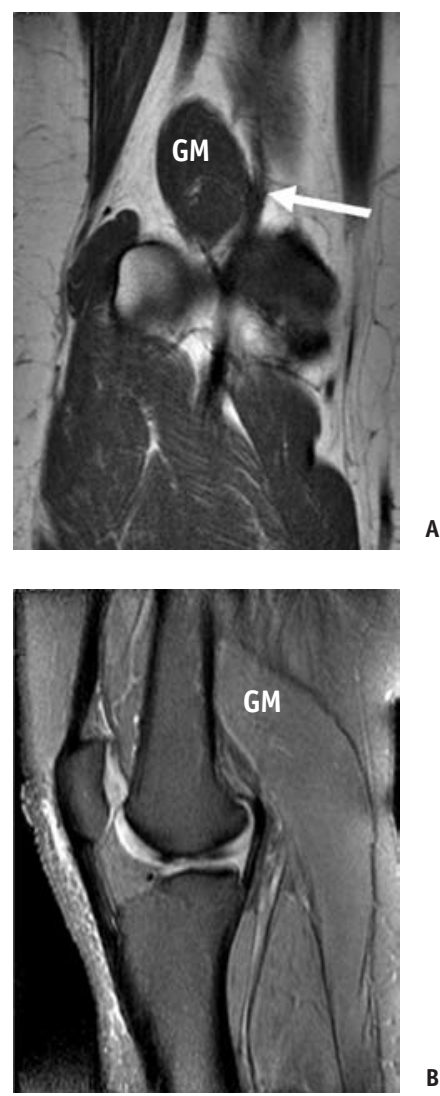

Figura 3 - Corte coronal FSE ponderada em T1 em plano posterior aos côndilos femorais (A) e corte sagital densidade de prótons em plano discretamente lateral à linha mediana (B). Na figura A, pode-se notar claramente o trajeto anormalmente lateralizado da cabeça medial do músculo gastrocnêmio (GM). A artéria poplítea tem trajeto levemente medianizado (seta). A figura B mostra a inserção alta e lateral do ventre medial do gastrocnêmio medial.
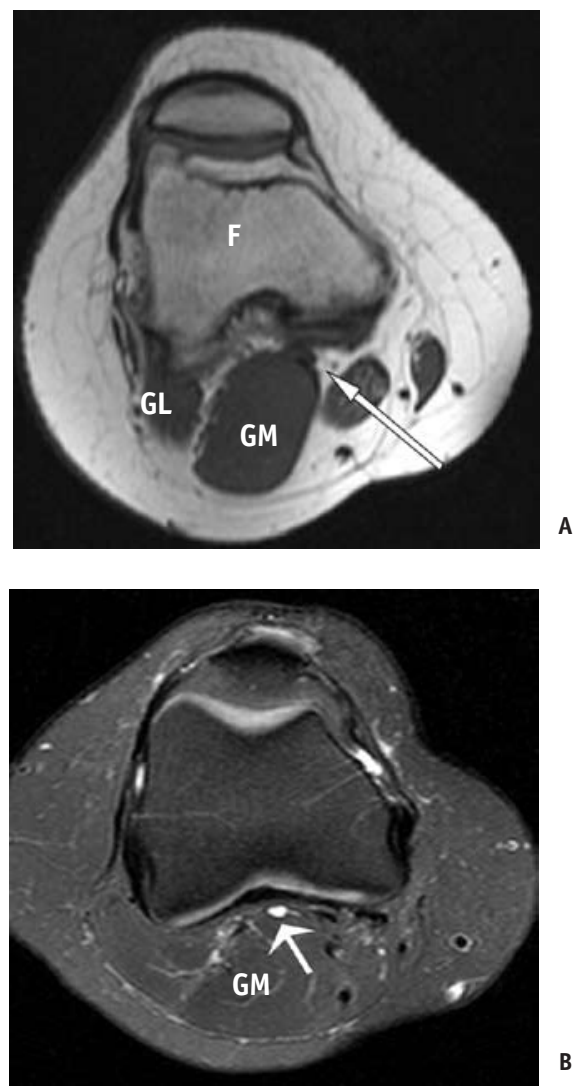

Figura 4 - Cortes axiais ponderados em T1 logo acima dos côndilos femorais (A) e em T2 com saturação de gordura em plano pouco abaixo de A. Em A evidenciase a artéria poplítea (seta), comprimida entre o fêmur (F) e o ventre medial do gastrocnêmio (GM). Nota-se também a cabeça lateral do gastrocnêmio (GL) inserindo-se normalmente no fêmur. Em B, destaca-se a perda do "flow-void" na artéria poplítea (seta curta) que se apresenta com hipersinal. 


\section{TRATAMENTO}

Devido à sua natureza progressiva, a síndrome do entrelaçamento da artéria poplítea deve ser diagnosticada e tratada precocemente. $\mathrm{O}$ procedimento cirúrgico com retirada do fator compressivo e eventual reconstrução arterial ou endarterectomia nos casos mais graves é o tratamento de escolha. Para isso, um planejamento cirúrgico baseado em imagens multiplanares e com a definição mais precisa sobre a etiologia da síndrome do entrelaçamento é aconselhável ${ }^{(7)}$.

\section{CONCLUSÃO}

A claudicação na perna pode ter várias etiologias, destacando-se a aterosclerose. Uma causa menos comum

\section{REFERÊNCIAS BIBLIOGRÁFICAS}

1. Macedo TA, Johnson CM, Hallet Jr JW, Breen JF: Popliteal artery entrapment syndrome: role of imaging in the diagnosis. AJR 181: 1259-65, 2003.

2. Elias DA, White LM, Rubenstein JD, Christakis M, Merchant $\mathrm{N}$ : Clinical Evaluation and MR imaging features of popliteal artery entrapment and cystic adventitial disease. AJR 180: 627-32, 2003.

3. Sperryn CW, Beningfield SJ, Immelman EJ: Functional entrapment of the popliteal artery. Australas Radiol 44: $121-4,2000$.

4. Erdoes LS, Devine JJ, Bernhard VM, Baker MR, Berman SS, Hunter GC: Popliteal vascular compression in a normal population. J Vasc Surg 20: 978-86, 1994.

5. Rosset E, Hartung O, Brunet C, et al.: Popliteal artery entrapment syndrome: anatomic and embryologic bases, diagnostic and therapeutic considerations following a series of 15 cases with a review of the literature. Surg Radiol Anat 17: 161-9, 1995. é a síndrome do entrelaçamento da artéria poplítea. Seu diagnóstico deve ser suspeitado na presença de sinais e sintomas de insuficiência arterial crônica progressiva em pacientes jovens, com massa muscular proeminente, e sem fatores de risco para aterosclerose. Diante dessa possibilidade, ultra-som Doppler pode caracterizar algumas alterações vasculares e anatômicas que corroborem com tal suspeita. Entretanto, na persistência da dúvida clínica, a ressonância magnética dirigida para o estudo da fossa poplítea a suas estruturas vasculares pode fornecer dados mais precisos sobre a etiologia da queixa clínica, além de facilitar o planejamento cirúrgico terapêutico.

6. Atila S, Akpek ET, Yucel C, Tali ET, Isik S: MR imaging and MR angiography in popliteal artery entrapment syndrome. Eur Radiol 8: 1025-9, 1998.

7. Levie LJ, Veller MG. Popliteal artery entrapment sybdrome: more comum tha previously recognized. J Vasc Surg 30: 587-98, 1990.

8. Foster BB, Houston JG, Machan LS, Doyle L: Comparison of two-dimensional time -of-flight dynamic magnetic resonance angiography with digital subtraction angiography in popliteal artery entrapment syndrome. Can Assoc Radiol J 48: 11-8, 1997.

9. Thomas NP, Jackson AM, Aichroth PM: Congenital abcense of the anterior cruciate ligament. A commom component of knee dysplasia. J Bone Joint Surg Br 67: 572-5, 1985.

10. Kaelin A, Hulin PH, Carlioz H: Congenital aplasia of the cruciate ligaments. A report of six cases. J Bone Joint Surg Br 68: 827-8, 1986.

11. Manner HM, Radler C, Ganger R, Grill F: Dysplasia of the cruciate ligaments: radiographic assessment and classification. J Bone Joint Surg 88: 130-7, 2006. 